\title{
EEG-based Brain-Computer Interfaces exploiting Steady-State Somatosensory-Evoked Potentials: A Literature Review
}

\author{
Jimmy Petit ${ }^{(1)}$, José Rouillard ${ }^{(1)}$ and François Cabestaing $(\mathbb{D}$ \\ Univ. Lille, CNRS, Centrale Lille, UMR 9189 CRIStAL, F-59000 Lille, France \\ E-mail: jimmy.petit@univ-lille.fr, jose.rouillard@univ-lille.fr, \\ francois.cabestaing@univ-lille.fr
}

September 2021

\begin{abstract}
.
A brain-computer interface aims to derive commands from the user's brain activity in order to relay them to an external device. To do so, it can either detect a spontaneous change in the mental state, in the so-called "active" BCIs, or a transient or sustained change in the brain response to an external stimulation, in "reactive" BCIs. In the latter, external stimuli are perceived by the user through a sensory channel, usually sight or hearing. When the stimulation is sustained and periodical, the brain response reaches an oscillatory steady-state that can be detected rather easily.

We focus our attention on EEG-based BCIs in which a periodical signal, either mechanical or electrical, stimulates the user skin. This type of stimulus elicits a steady-state response of the somatosensory system that can be detected in the recorded EEG. The oscillatory and phase-locked voltage component characterising this response is called a steady-state somatosensory-evoked potential (SSSEP).

It has been shown that the amplitude of the SSSEP is modulated by specific mental tasks, for instance when the user focuses their attention or not to the somatosensory stimulation, allowing the translation of this variation into a command. Actually, SSSEP-based BCIs may benefit from straightforward analysis techniques of EEG signals, like reactive BCIs, while allowing self-paced interaction, like active BCIs.

In this paper, we present a survey of scientific literature related to EEG-based BCI exploiting SSSEP. Firstly, we endeavour to describe the main characteristics of SSSEPs and the calibration techniques that allow the tuning of stimulation in order to maximise their amplitude. Secondly, we present the signal processing and data classification algorithms implemented by authors in order to elaborate commands in their SSSEP-based BCIs, as well as the classification performance that they evaluated on user experiments.
\end{abstract}

Keywords: BCI, Steady-State Somatosensory-Evoked Potentials, SSSEP, EEG, Signal Processing

Submitted to: J. Neural Eng. 


\section{Introduction}

In EEG-based brain-computer interfaces (BCI) studies, many different paradigms have been proposed, exploiting various markers of mental activity. These markers detect either a volitional modulation of the ongoing brain activity or a specific event elicited by an external stimulus. In EEG signals, the specific brain activity elicited by a stimulation is detected as a small voltage variation immediately following it, called an event-related potential or evoked potential.

The most known evoked potentials used in BCIs are P300 and steady-state visually-evoked potentials (SSVEP). SSVEPs are quite easy to use as they allow the control of an application by detecting oscillations in EEG signals recorded over the occipital cortex, while the user is looking at visual stimuli flashing at different frequencies. In the same way, periodical beeping sounds, periodical mechanical stimulation of the skin, or electrical stimulation of skin nerves at a constant frequency, elicit steady-state oscillating brain responses, measured respectively by steady-state auditory-evoked potentials (SSAEP) and steady-state somatosensory-evoked potentials (SSSEP).

When the external mechanical or electrical stimulation is applied to the skin, it activates the somatosensory system. In this case, the evoked potential is referred to as a somatosensory-evoked potential (SEP). The stimulation can be either transient or sustained and periodic. After a transient stimulation, specific subcomponents - deflection or inflexion - can be observed in the electrical signal before the brain returns to an "idle" state. Thus, SEP signal analysis is usually performed in the time domain [14]. With a sustained and periodic stimulation, when the time interval between two successive stimuli is too small, the somatosensory system cannot come back to an idle state in between them [28]. Thus, the SEP signal analysis is preferably performed in the frequency domain [15]. More precisely, an increase of power can usually be measured in the SEP signal at the frequency of stimulation or its harmonics. SEPs evoked by a sustained and periodic stimulation are referred to as steady-state somatosensory-evoked potentials (SSSEP). In the rest of the article, a mechanical stimulation of the skin or an electrical stimulation of skin nerves, delivered at a constant frequency, will be referred to as a periodical somatosensory stimulus.
Since the mid-1960s, SEPs have been used as a monitoring tool during neurosurgical procedures or spinal surgery [45]. For example, by stimulating the posterior tibial nerve and by monitoring the SEPs in the somatosensory cortex during spinal surgery, early surgery-related damages to the motor capacity of the patient can be detected [30]. SSSEP have also been used in different clinical applications, for instance to measure the tactile acuity of amputees [41] or as a marker for monitoring cortical processes resulting from a nociceptive and non-nociceptive somatosensory input [11].

Figure 1 shows an histogram of publications per year reflecting the dynamics of SSSEP-oriented research (from the PubMed database, see section 2). After a narrow and centred peak in the number of publications in 2013, the research activity decreases year by year. Despite this apparent lack of interest from the BCI community, we consider that this field is still relevant to study. Indeed, a SSSEP-based BCI exploits a communication channel that is barely used by motor-impaired people. Therefore, we consider that from the user's point of view, even a small benefit provided by a SSSEP-based BCI using a lost interaction modality is welcome, since the system does not monopolize their sight or hearing.

Additionally, eye fatigue often occurs during the use of SSVEP-based BCI systems and major SSAEPbased BCI systems suffer from low accuracy [34]. Interesting ideas lead to actionable BCIs for certain types of users, for whom audio and visual stimulations cannot be used. For instance, Giabbiconi et al measured, in 2004, that the amplitudes of elicited SSSEPs were modulated by the spatial attention of

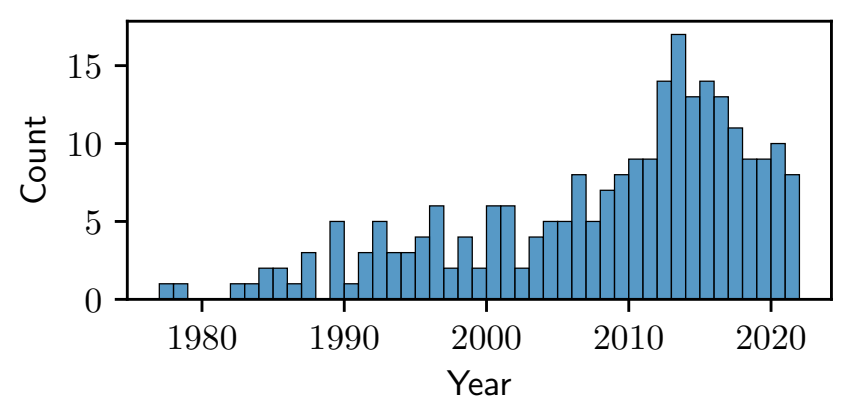

Figure 1. Histogram of the number of publications per year from the PubMed database with the keywords "steady-state somatosensory" in "all fields" the 24th of August 2021. 
the subject [15]. It appears that by focusing their attention on the stimulation, the subject could increase the amplitude of SSSEPs.

Extensive literature reviews on non-stationary evoked-potentials, either visually, auditory or somatosensory-evoked, have already been published, for instance [4]. Moreover, a recent literature review on haptics and/or tactile BCIs has also been recently published [13]. Therefore, we limited the scope of our review to research studies in which periodical somatosensory stimuli are used to elicit SSSEP measured by EEG. In section 2 we present how we identified, screened and included articles according to the PRISMA (preferred reporting items for systematic reviews and meta-analyses) methodology [23]. We compare our set of studies to the different references from identified state-of-the-arts while presenting our inclusion and exclusion criteria.

In section 3, we define some specific characteristics of SSSEPs useful for BCI. In order to use SSSEP variations as robust markers in a $\mathrm{BCI}$, one or several optimal stimulation frequencies must be determined. Thus, the notions of frequency of stimulation (FOS) and resonance-like frequencies are presented, as well as techniques for determining user-specific frequencies. First synoptical tables (tables 1a and 1b) presents how the selected studies performed a determination of user-specific frequencies, as well as their main results: stimulation location, type of vibration, used FOS or range of FOS, etc.

Section 4 presents the algorithms and performances of SSSEP-based BCIs from the literature. A second pair of tables (tables $2 \mathrm{a}$ and $2 \mathrm{~b}$ ) presents the algorithms and the obtained performances. Finally, before the conclusion, we summarize the main results of this SSSEP-based BCI literature review.

\section{Methodology}

In August 2021, we started to review the literature on SSSEPs and SSSEP-based BCIs in accordance to the PRISMA methodology. Figure 2 presents our article selection flowchart. More details about the identification, screening and inclusion stages follow.

\subsection{Identification stage}

A search on the PubMed database was performed on the $24^{\text {th }}$ of August 2021 with the keywords "steadystate somatosensory" in "all fields". This search brought to light 242 items.

Inputs from three state-of-the-art articles were also considered. The first one, chronologically, elaborates on BCIs leveraging the sense of touch [12]. Its scope is broader than ours, since it also includes BCI studies that use a mechanical stimulation of the skin to provide a feedback to the user, or to elicit P300 transient SEPs. The second state-of-the-art article is clearly focused on SSSEP and SSSEP-based BCIs [3]. It appeared relevant to include both articles in the present literature review for two reasons:

- Even though the second state-of-the-art article can be considered conceptually included in the perimeter of the first one, it remains interesting because it analyses an additional two-year of insights on this scientific domain.

- Only one article reference is presented in both state-of-the-art articles, among the 35 presented in [12] and the 37 presented in [3].

A recent survey on the use of haptic feedback for BCI [13] was also analysed, since a short section is dedicated to SSSEP-based BCIs.

\subsection{Screening stage}

We considered only articles fully written in English, in which measurements were realised using EEG, and presenting either a study of human specific SSSEP characteristics or an SSSEP-based BCI.

The abstracts of the first 124 items, ordered by "best match", were thoroughly analysed. The analysis showed that 2 items were not research articles, 45 items were not related to SSSEP, 28 used different measurements than EEG, and 8 did not provide insights on an SSSEP-based BCI or a determination of user-specific frequencies. The 38 remaining items were read entirely to assess their eligibility. This second analysis showed that 26 items could not be included since they were describing neither a determination of user-specific frequencies nor an SSSEP-based BCI.

\subsection{Inclusion stage}

12 articles were included as a result of the screening stage. 5 additional articles, identified during previous searches or corresponding to bibliographical entries of the 12 selected articles, were also included. Finally, 7 additional articles referenced in the state-of-the-art articles were included, ending up with a total of 24 articles studied in our review.

17 articles describe a stimulation, recording and processing procedure that allows the estimation of user-specific characteristics of SSSEPs. These studies are presented and analysed in section 3 . Section 4 is devoted to the presentation of 17 articles that describe an SSSEP-based BCI. 10 studies, that deal with both topics, are presented in the two sections from different points of view. 


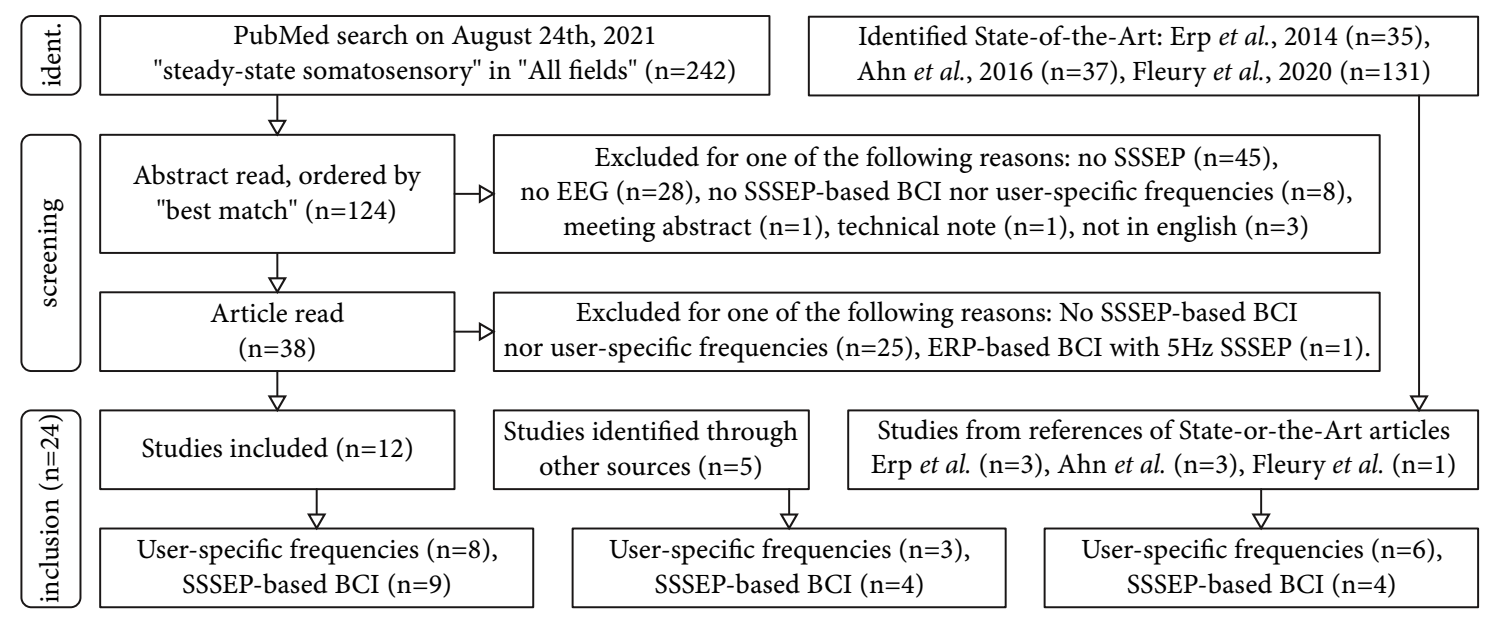

Figure 2. PRISMA-based article inclusion: identification, screening and inclusion stages.

\section{Effects of stimulus characteristics on the evoked potential}

\subsection{Methods to elicit an SSSEP}

Historically, in most studies aiming to study SEPs, the latter were elicited by electrical stimulation of peripheral nerves [28, 40, 29]. For instance, a correctly adjusted current flowing between two electrodes placed over the median nerve near the wrist can elicit an SEP. Indeed, the intensity of current pulses is increased until they produce tiny twitches of the thenar muscle, located on the hand palm at the base of the thumb, and simultaneously elicit SEPs [28].

Beside electrophysiological studies, this method of stimulation offers great tools to clinicians for monitoring patient state, for example during delicate spinal chord surgery [30]. However, electrical stimulation of peripheral nerves is reported as unpleasant and elicits SEPs with low amplitude [29, 40]. Therefore, efforts have been made to switch to mechanical stimulation, especially in the context of brain-computer interfacing where the system must be as comfortable to use as possible during long periods.

The nature of the mechanical stimulation is the first aspect to consider when designing an SSSEPbased BCI. In order to elicit an SSSEP, the mechanical stimulation must be sustained and periodical, and several techniques can be used to that end. The first one consists in producing short mechanical pulses, for instance with a moving pin, like the ones used in dot matrix printer heads, in contact with the skin [24]. The shape of this type of stimulation pattern is considered to be identical to the shape of the electrical signal driving the pin, usually a low frequency square wave with a duty cycle of $50 \%$. However, in order to elicit SSSEPs with higher amplitudes, Pacinian corpuscles can be stimulated more efficiently. Pacinian corpuscles are one of the four types of mechanoreceptors of the human skin. Their maximum sensitivity is for a mechanical vibration with a frequency between 200 and $250 \mathrm{~Hz}$ [38]. This frequency is way too high to elicit an SSSEP that can be measured on the cortex. Therefore, the mechanical stimulation is actually delivered at a carrier frequency around $250 \mathrm{~Hz}$, but with an amplitude modulated at a much lower frequency [43, 7]. In this case, SSSEPs are elicited at the frequency of the modulation, not of the carrier. This technique is referred to as vibro-tactile stimulation. The amplitude can be modulated either by a square or a sinusoidal function.

In this article, we use the terms short mechanical pulses and vibro-tactile stimulation, both introduced by Müller-Putz et al in 2001 [24]. It is noteworthy to mention that electrical stimulation patterns can also be amplitude-modulated. In this case, similarly to vibro-tactile stimulation, the stimulation current is a high-frequency carrier current whose amplitude is modulated by a low-frequency sinusoidal signal [29].

\subsection{SEP and SSSEP characteristics}

The frequency of stimulation (FOS) is the frequency of the sustained and periodical somatosensory stimulation eliciting the SSSEP. More precisely, it is either the frequency of short electrical or mechanical pulses or the frequency modulating a carrier stimulation. In the frequency domain, the SSSEP is characterized by a high value, at the FOS, of the power or amplitude of the measured electrical activity.

The resonance-like frequency is a term introduced by Müller-Putz et al in 2001 [24]. It is defined as the particular FOS that elicits an SSSEP with the highest amplitude or signal-to-noise ratio, 
assuming that the stimulation amplitude remains constant. When the SSSEP amplitude vs. frequency curve shows several local maxima, the resonance-like frequency is not unique. When this curve does not show a sharp maximum, the resonance-like frequency is sometimes defined as a frequency band [24]. Usually, in an SSSEP-based BCI, at least two FOS are required, one of them being the resonance-like frequency. Other FOS are selected at other local maxima of the amplitude vs. frequency curve if they exist, provided that they are different enough from the resonancelike frequency. To achieve this, keeping a minimum difference of $4 \mathrm{~Hz}[6,33]$ or $5 \mathrm{~Hz}[26,1]$ between each FOS is a common practice.

When talking about latency several aspects must be taken into consideration. Strictly speaking, when considering the whole causal chain, i.e. from stimulation to human to measurement, the latency is the time between the stimulus onset (the cause) and the SEP appearance in the processed electrical signal (the effect). Hence, the latency encompasses several delays:

- the transport time that is required to transfer the stimulation-related information from the skin to the brain.

- the cortical processing time that encompasses all the delays introduced by following neural pathways within the cortex before SEP appearance.

- the hardware delay, that can be caused by several factors, such as transfer delays in pipeline processing stages after signal sampling, or drifts resulting from lost or artificially inserted digital signal samples in asynchronous communication channels.

- the delays that can be introduced by signal processing techniques, such as narrow bandwidth signal filtering.

These components of the latency cannot be measured separately in practice. For example, the transport time and the cortical processing time are hardly differentiable. A hardware delay, from the computer to the stimulation device, has been measured in a study conducted by Pokorny et al in 2014 [32]. They concluded that this delay, a few hundreds of microseconds, can be neglected compared to other delays. Finally, delays caused by signal filtering have been known for a long time. The interested reader can refer to an article of De Cheveigné et al in which the authors extensively discuss the implication of filters, either hardware and software, and also suggest delay compensation techniques [9].

In this article, we use the term total latency to denote the sum of all delays. In contrast, we use the term apparent latency, defined by Regan in $[36,37]$, to characterize only the physiological part of the total latency. For Regan, the apparent latency is the first derivative of the SSSEP phase with respect to the stimulus frequency, i.e. the slope of an SSSEP frequency vs. phase plot. One must keep in mind that even the apparent latency cannot be interpreted rigorously from a neurological point of view. Indeed, a long apparent latency can be caused either by a long transport time followed by a short cortical processing time or by the opposite.

From the human-computer interaction (HCI) point of view, minimizing the total latency or at least keeping it constantly below a threshold is of major importance. Indeed, in the context of real-time interaction, the total latency can also be defined as the minimum time it takes for the BCI to detect a user's intention after the stimulation onset.

The phase difference is simply the time difference between similar zero crossings (i.e. with the same slope sign) of the SEP on the one hand and of the stimulation signal on the other hand, times the FOS and a constant to get an angle-like quantity. It should not be confused with the phase shift, as defined by Regan in [37], that is the sum of the phase difference and of the apparent latency, also expressed as an angle.

A schematic definition of apparent latency and phase difference characterizing the onset of a theoretical SSSEP is presented in figure 3.

The time-to-stationarity can be defined as the duration of the transient response to the periodical somatosensory stimulus, that starts with transient somatosensory-evoked potentials before reaching a steady-state response at the frequency of stimulation. It is assumed that the stimulation remains constant as well as the subject's mental activity, for instance that there is no attention focusing or stimulation triggered mental action. Brickwedde et al measured a time-tostationarity of $500 \mathrm{~ms}$ for a SSSEP evoked by a $20 \mathrm{~Hz}$ vibration [8]. Figure 4 shows the stabilisation of an SEP into an SSSEP measured by Brickwedde et al [8].

\subsection{Determination of user-specific frequencies}

When the skin is stimulated by a periodical somatosensory stimulus, the elicited SSSEP is characterized by measuring the electrical brain activity at the FOS. Many studies have shown that the amplitude and signal-to-noise ratio of the SSSEP are highly dependent on the FOS $[5,33]$. Thus, for each subject and each stimulation position, a specific screening procedure is often performed to estimate the amplitude or signal-to-noise ratio of the SSSEP with respect to the FOS. We refer to this procedure as the tuning curves estimation.

During each trial of the tuning curves estimation procedure, a mechanical stimulation is applied on 
a)

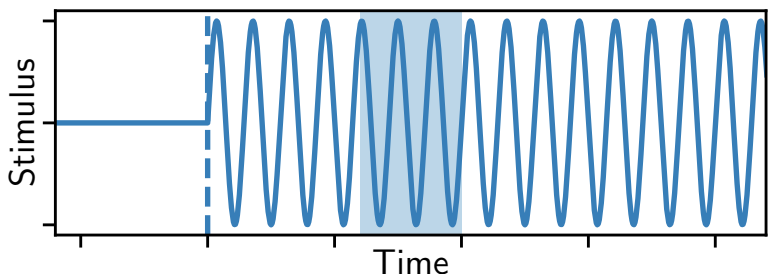

b)

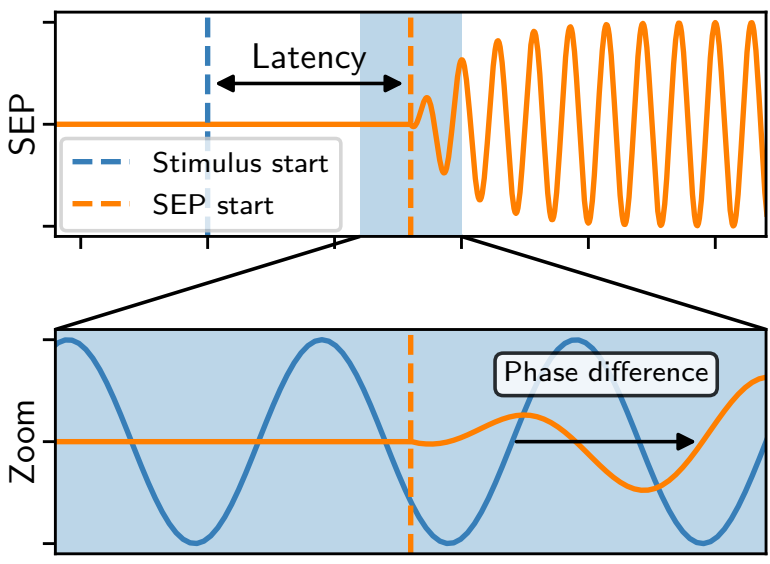

Figure 3. Temporal characteristics of an theoretical SEP reaching its steady-state. (a) The "stimulus" plot is the representation of the periodical somatosensory stimulus amplitude vs. time, considered here as a windowed sine function. (b) Representation of an ideal SEP elicited by the stimulus, i.e. an amplitude modulated sine function without noise. (c) Zoom on the plots (a) and (b) around the beginning of the SEP. It shows the phase difference between the SEP and the stimulus.

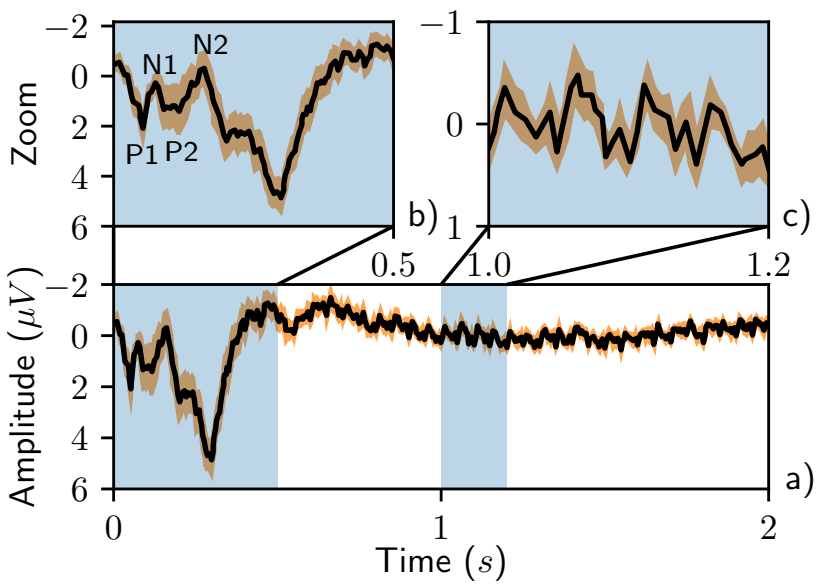

Figure 4. Temporal characteristics of an SEP reaching its steady-state. (a) Grand average over 14 subjects and $40 \mathrm{~min}$ of $20 \mathrm{~Hz}$ stimulus train on the right index finger for $2 \mathrm{~s}$, with a $5 \mathrm{~s}$ inter-trial interval. (b) Zoom on the first half second of the grand average, clear component of the SEP are annotated. (c) Zoom on an SSSEP-established period, 4 periods of the $20 \mathrm{~Hz}-$ SSSEP are well visible over $0.2 \mathrm{~s}$ of signal. All data is presented from $\mathrm{CP} 1$ and as mean \pm SEM. Figure reproduced with the appreciated authorisation of the authors from [8]. a given part of the body, with a given FOS, and sometimes for a given duration or shape of amplitude modulation [33]. The tested frequencies, as well as other tested parameters, are usually randomized during the successive trials. Each FOS or stimulation parameter is tested several times, to average the results and increase the measurement precision. This procedure yields one or several tuning curves, that characterize the relationship between the frequency, duration and shape of the stimulation, and the amplitude, power, or signal-to-noise ratio of the SSSEP.

The following two tables summarize the literature describing these procedures. Table 1a presents the methodology of each study regarding the determination of user-specific frequencies. Table $1 \mathrm{~b}$ presents the results of studies introduced in table 1a. The first 3 columns, present in the two tables, are used as an index: name of the first author, paper reference and stimulation location. The five following columns in table 1a present the methodology, in order: hardware vibrator (Vibrator), surface of the contact area of the vibrator head with the skin (Size), stimulation type and carrier frequency (Stimulation), range and number of tested FOS (FOS or FOS range), and the number of subjects (Subj.) involved in the experiment. The three last columns of table $1 \mathrm{~b}$ present the main results. The column RLF reports the resonance-like frequency. The next column TC mention if the tuning curves from the determination of user-specific frequencies were reported in their completeness in the study and the last column provides supplementary information when needed.

\subsection{Determination of user-specific frequencies in the literature: main results and comments}

In the seminal article published in 1992, Snyder introduces the main physiological characteristics of SSSEPs such as apparent latency, dependence of amplitude to FOS, and spatial location of the stimulusinduced neuronal activity [40]. An EEG setup with 16 channels was used in his experiment, with electrodes over the scalp at locations following the international 10-20 system. The subjects rested their hand (left or right) on top of a rigid plastic spherical shell, with a diameter of 8 centimetres, acting as the vibrator head. Vibro-tactile stimulation, with a carrier frequency of $128 \mathrm{~Hz}$, was used in this experiment. At the maximum amplitude, the vibration head provided a thrust of about 10 Newtons, producing for some subjects a feeling of "rising hand". Different FOS were tested by Snyder:

- 2 and $3 \mathrm{~Hz}$ FOS on 4 subjects;

- $5,7,11,17,26,40 \mathrm{~Hz}$ on 17 subjects; 


\begin{tabular}{|c|c|c|c|c|c|c|c|}
\hline First author & Ref. & $\begin{array}{l}\text { Stimulation } \\
\text { Location }\end{array}$ & Vibrator & $\begin{array}{l}\text { Size } \\
\left(\mathrm{cm}^{2}\right)\end{array}$ & Stimulation & $\begin{array}{c}\text { FOS or FOS range } \\
\quad(\# \text { of FOS })\end{array}$ & Subj. \\
\hline Snyder & {$[40]$} & & SS & $100.5^{*}$ & $\begin{array}{l}\text { Vibration } \\
(128 \mathrm{~Hz})\end{array}$ & $\begin{array}{c}2 \& 3 \\
5 \text { to } 40(6) \\
25 \& 27\end{array}$ & $\begin{array}{c}4 \\
17 \\
13\end{array}$ \\
\hline Tobimatsu & {$[43]$} & & SS & $127^{*}$ & $\begin{array}{l}\text { Vibration } \\
(128 \mathrm{~Hz})\end{array}$ & 5 to $30(9)$ & 10 \\
\hline Tobimatsu & {$[44]$} & & SS & $127^{*}$ & $\begin{array}{l}\text { Vibration } \\
(128 \mathrm{~Hz})\end{array}$ & 17 to $30(6)$ & 8 \\
\hline $\begin{array}{l}\text { Müller-Putz } \\
\text { Wang }\end{array}$ & $\begin{array}{l}{[26]} \\
{[46]}\end{array}$ & & $\begin{array}{l}\mathrm{NP} \\
\mathrm{KoP}\end{array}$ & $\begin{array}{l}0.03 \\
\mathrm{~N} / \mathrm{A}\end{array}$ & $\begin{array}{l}\text { Pulses } \\
\text { N/A }\end{array}$ & $\begin{array}{l}17 \text { to } 35(10) \\
5 \text { to } 29(25)\end{array}$ & $\begin{array}{c}5 \\
5 ?\end{array}$ \\
\hline Breitwieser & {$[7]$} & & $\mathrm{C} 2$ & 0.45 & $\begin{array}{l}\text { Vibration } \\
(200 \mathrm{~Hz})\end{array}$ & 13 to 35 (12) & 14 \\
\hline Ahn & {$[2]$} & & $\mathrm{LRA}^{?}$ & 0.28 & $\mathrm{~N} / \mathrm{A}$ & 21 to $25(5)$ & 8 \\
\hline Ahn & {$[1]$} & & LRA? $^{2}$ & 0.79 & $\mathrm{~N} / \mathrm{A}$ & 21 to $25(5)$ & 16 \\
\hline Breitwieser & {$[5]$} & & $\mathrm{C} 2$ & 0.45 & $\begin{array}{l}\text { Vibration } \\
(200 \mathrm{~Hz})\end{array}$ & 17 to $35(10)$ & 9 \\
\hline Pokorny & {$[32]$} & & $\mathrm{C} 2$ & 0.45 & $\begin{array}{l}\text { Vibration } \\
(200 \mathrm{~Hz})\end{array}$ & 14 to $32(7)$ & 1 \\
\hline Müller-Putz & {$[24]$} & & $\mathrm{NP}$ & 0.03 & Pulses & 17 to $31(8)$ & 10 \\
\hline Breitwieser & {$[6]$} & & $\mathrm{C} 2$ & 0.45 & $\begin{array}{l}\text { Vibration } \\
(237 \mathrm{~Hz})\end{array}$ & 17 to $35(10)$ & 13 \\
\hline Pokorny & {$[33]$} & & $\mathrm{C} 2$ & 0.45 & $\begin{array}{l}\text { Vibration } \\
(237 \mathrm{~Hz}) \\
\end{array}$ & 17 to $35(10)$ & 14 \\
\hline Kim & {$[19]$} & & ERM & 0.79 & Pulses $^{? \dagger}$ & 13 to $35(12)$ & $4 ?$ \\
\hline Kim & {$[20]$} & & ERM & 0.79 & Pulses ${ }^{? \dagger}$ & 13 to $35(12)$ & $5 ?$ \\
\hline Kim & {$[21]$} & & ERM & 0.79 & Pulses $^{? \dagger}$ & 13 to 35 (12) & 12 \\
\hline Kee & {$[17]$} & & ERM & 0.79 & Pulses ${ }^{? \dagger}$ & 13 to 33 (11) & 5 \\
\hline
\end{tabular}

Table 1a. User-specific frequencies identifications: Methodology. Legend and Acronyms: Vibration: vibro-tactile stimulation (carrier frequency at X Hz); Pulses: short mechanical pulses; FOS: frequency of stimulation; Subj.: number of subjects; SS: spherical shell; NP: dot matrix needle printer; KoP: Knock-out Pin; C2: C-2 tactor; LRA: Linear Resonant Actuator; ERM: Eccentric Rotating Mass; N/A: not available; *: skin contact assumed to be perfect; ${ }^{\dagger}$ : N/A but likely as in [24], i.e. short mechanical pulses; ?: unclear or ambiguous.

- and 25 and $27 \mathrm{~Hz}$ on 13 subjects.

Firstly, Snyder reports that the SSSEP appeared in the hemisphere contralateral to the stimulated hand on parts of the primary and secondary somatosensory cortex, and on the primary motor cortex. The same locations, when reported, were determined in almost all studies listed in tables 1a and 1b. Besides, Snyders' results show that FOS at $26 \mathrm{~Hz}$ and $40 \mathrm{~Hz}$ seem to produce SSSEPs with higher signal-to-noise ratios than other FOS. Among those two FOS, the author observed that the amplitude of SSSEP at $26 \mathrm{~Hz}$ was greater.

An approximation of the apparent latency, using an estimation of the EEG background noise from the averaged evoked responses, was also calculated. This was performed on a combination of 19 datasets: 13 subjects each-one producing 2 datasets, one per hand, 7 datasets were rejected. The estimated distribution of the apparent latency, over the FOS at 25, 26 and
$27 \mathrm{~Hz}$, has a mean of $58.6 \mathrm{~ms}$ and a standard deviation of $14.6 \mathrm{~ms}$.

Regarding the resonance-like frequency, in the articles listed in table 1b, two trends can be observed. Tuning curves, i.e. amplitude of SSSEP vs. FOS, show either a rather flat maximum centred at $27 \mathrm{~Hz}[24$, $33,6]$ or more pronounced local maxima centred on lower frequencies such as $17 \mathrm{~Hz}$ or $21 \mathrm{~Hz}$ [46, 21]. The first trend confirms the results from [40] whereas the other trend confirms the results from [43, 44]. Other determination of user-specific frequencies did not yield tuning curves similar to either trend, such as the one in the article of Breitwieser et al in 2011, that yielded tuning curves with a rather flat maximum centred on a FOS varying from 21 to $35 \mathrm{~Hz}$ [7].

In the articles presented in tables $1 \mathrm{a}$ and $1 \mathrm{~b}$, studies also seem to show that FOS below $20 \mathrm{~Hz}$ rarely produce SSSEPs with high amplitudes or signal-to- 


\begin{tabular}{|c|c|c|c|c|}
\hline First author & Ref. & $\begin{array}{l}\text { Stimulation } \\
\text { Location }\end{array}$ & RLF (body part) & TC Observations \\
\hline Snyder & {$[40]$} & & $26 \& 40$ & $\begin{array}{l}40 \mathrm{~Hz} \text { SSSEP have small amplitude but similar } \\
\text { Yes signal-to-noise ratio than } 26 \mathrm{~Hz}-\mathrm{SSSEP} \text {. Both hands } \\
\text { were stimulated at } 25 \& 27 \mathrm{~Hz} \text {. }\end{array}$ \\
\hline Tobimatsu & {$[43]$} & & 21 & Yes \\
\hline Tobimatsu & {$[44]$} & & 21 & $\begin{array}{l}\text { Identified RLF for } 6 \text { out of } 8 \text { subjects. Low-to-none } \\
\text { impact of the frequency on the sole-elicited SSSEP. } \\
\text { Ymplitude of sole-elicited SSSEP are roughly twice } \\
\text { lower than hand-elicited SSSEP. }\end{array}$ \\
\hline Müller-Putz & {$[26]$} & & 25 to 31 & No \\
\hline Wang & {$[46]$} & & $\mathrm{N} / \mathrm{A}$ & $\begin{array}{l}\text { Tuning curve presented for } 1 \text { subject out of } 5 \\
\text { subjects, grand average not available. }\end{array}$ \\
\hline Breitwieser & {$[7]$} & & $21 \& 23$ & No Identified RLF for 7 out of 14 subjects. \\
\hline Ahn & {$[2]$} & & $\mathrm{N} / \mathrm{A}$ & No \\
\hline Ahn & {$[1]$} & & $22 \& 23$ & No Identified RLF for 10 out of 14 subjects. \\
\hline Breitwieser & {$[5]$} & & $\mathrm{N} / \mathrm{A}$ & $\begin{array}{l}\text { The TC for each subject highlight how important the } \\
\text { inter-subject variability is. Each subject performed } \\
\text { two sessions within several weeks (Mean: } 28 \text { day, Std: } \\
\text { 17.5): the TC between sessions are very similar. }\end{array}$ \\
\hline Pokorny & {$[32]$} & & 20 & Yes Irrespective of the wrist. \\
\hline Müller-Putz & {$[24]$} & & 27 (Left index) & $\begin{array}{l}\text { Low-to-none influence of the frequency on the SSSEP } \\
\text { for right index. }\end{array}$ \\
\hline Breitwieser & {$[6]$} & & 23 to 27 & $\begin{array}{l}\text { No Identified RLF for } 9 / 13 \text { subjects (right index) and } \\
8 / 13 \text { subjects (left index). }\end{array}$ \\
\hline Pokorny & {$[33]$} & & 27 & Yes Irrespective of the index fingertip. \\
\hline Kim & {$[19]$} & & $\begin{array}{l}17 \text { to } 23 \text { (index) } \\
27 \text { to } 31 \text { (toe) }\end{array}$ & No Irrespective of the index. \\
\hline Kim & {$[20]$} & & $\begin{array}{l}13 \text { to } 25 \text { (index) } \\
29 \text { (toe) }\end{array}$ & No Irrespective of the index. \\
\hline Kim & {$[21]$} & & $\begin{array}{l}17 \text { (index) } \\
27 \text { (toe) }\end{array}$ & No Irrespective of the index. \\
\hline Kee & {$[17]$} & & 13 to 29 & No Irrespective of the toe. Broad distribution of RLF. \\
\hline
\end{tabular}

Table 1b. User-specific frequencies identifications: Results. Legend and Acronyms: RLF: resonance-like frequency; TC: tuning curve; N/A: not available.

noise ratios. However, this might be caused by an averaging effect, since in rare subjects, it is useful to test nevertheless these low FOS in the determination of user-specific frequencies procedure, as shown in one article of Breitwieser et al [5]. In their study, they determined the user-specific FOS for all fingertips of the right hand for nine subjects. They used a vibrotactile stimulation with a carrier frequency at $200 \mathrm{~Hz}$. Each subject performed two sessions within weeks (mean 28 days, standard deviation 17.5 days). Six subjects out of nine had one resonance-like frequency below $20 \mathrm{~Hz}$ during one session, for at least one finger. Besides, the results remained rather stable between the two sessions; an ANOVA for repeated measure substantiates this observation. Thus, it seems relevant to assess low frequencies of stimulation as well for determining user-specific frequencies.

Finally, it is noteworthy that in the works of Kim et al, several subjects had already tested a BCI before taking up the main experiment $[19,20$, 21]. Therefore, across these papers, the resonance-like frequency can be biased if the same subject performed a similar screening procedure in several successive experiments. Indeed, we know from the work of Breitwieser et al that the resonance-like frequency tends to be stable through time [5]. Their work 
confirms stability for weeks, but for longer periods this property remains unknown.

It should also be noted that, to the best of our knowledge, the influence of the stimulation location on the total latency has not been reported in the literature.

3.4.1. Force and pressure of stimulation. The threshold of human skin sensitivity to mechanical stimulation has been quite studied and known for decades [47]. However, the influence of the intensity of the stimulation on the amplitude of the SSSEP remains unclear. The work of Tobimatsu et al in 1999 provides some insights on this question [43]. However, more recent studies in table $1 \mathrm{a}$ and $1 \mathrm{~b}$ tend to ignore it.

Tobimatsu et al investigated the impact of the stimulation force, measured in Newtons $(\mathrm{N})$, on the amplitude of the SSSEP. They tested different forces, from 0.001 to $0.1 \mathrm{~N}$, for the same FOS at $21 \mathrm{~Hz}$. In the frequency domain, they observed that the mean amplitude of the SSSEP component at the FOS linearly increases with the logarithm of the force up to a plateau, reached at $0.05 \mathrm{~N}$. This trend seems to hold also for the SSSEP amplitude measured at the second harmonic of the FOS, i.e. frequency equal to twice the FOS, even though much smaller.

However, for further investigation, the chosen stimulation intensity metric, i.e. the force, can be discussed. Indeed, the force controls directly the acceleration of the stimulation pin or head, but it does not take into account the contact surface between this device and the skin. For future studies investigating this influence, we think that it is advisable to use the force as metric, in order to have a comparison point with previous studies, but also the pressure exerted on the skin. This would help taking into account the specificity of the stimulation pin or head, which is important as demonstrated by the variety of devices described by the columns "Vibrator" and "Size" of table 1a. The lack of specificity of the force metric, in fact, may explain a part of the variability observed in the trends mentioned above.

3.4.2. Harmonics of the frequency of stimulation. Few studies assess the presence of harmonics of the FOS in the frequency analysis of SSSEPs. In 1996, Noss et al elicited SSSEPs with an amplitude modulated electrical stimulation at $200 \mathrm{~Hz}$ [29]. The modulation signal was a pure sine function, i.e. with no harmonic content at multiples of the fundamental frequency. However, peaks higher than the baseline were detected in the power spectrum of the SSSEP at frequencies corresponding to harmonics of the FOS. This, according to the authors, reveals a pronounced non-linearity of the transfer function of the somatosensory system.

In 1999, Tobimatsu et al also performed an harmonic analysis of SSSEPs, but for a mechanical vibro-tactile stimulation [43]. The carrier frequency at $128 \mathrm{~Hz}$ was modulated by a sinusoidal signal at frequencies ranging from 5 to $30 \mathrm{~Hz}$. They also report that a peak at twice the FOS can be detected in the power spectrum of the SSSEP, although smaller than the peak at the FOS. What is interesting though, is that the height of the peak at twice the FOS monotonically decreases as the FOS increases.

In fact, a similar phenomenon had already been mentioned by Snyder in 1992. We recall that in his experiments a vibro-tactile stimulation with a carrier at $128 \mathrm{~Hz}$ modulated by a sinusoidal FOS was used. Snyder observed that in the power spectrum of SSSEPs elicited by a FOS greater that $11 \mathrm{~Hz}$, the maximum power was localized at the FOS and almost no power increase was detected around its harmonics. On the other hand, in SSSEPs elicited by a FOS lower than $7 \mathrm{~Hz}$, the power increase at twice the FOS was almost as important as at the FOS itself.

It is noteworthy to mention that in the case of vibro-tactile stimulation, if the study of harmonics is considered, the modulation signal should be sinusoidal. Indeed, a modulation signal with a square shape inherently contains the FOS as well as its odd harmonics, but not the even ones. Thus, the second harmonic of the FOS is not present in the vibro-tactile stimulation and therefore cannot produce a response of the somatosensory perception chain. For instance, Breitwieser et al highlighted that no power increase could be found at the second harmonic of a FOS equal to $23 \mathrm{~Hz}$, when using a square modulation signal of a $200 \mathrm{~Hz}$ carrier frequency [7].

To summarise, in this section we reviewed research studies in which user-specific frequencies of stimulation are determined. We presented SEP and SSSEP characteristics, and then discussed techniques that aim to determine resonance-like frequencies. SSSEP's amplitude vs. FOS response curve is strongly userdependant, sometimes flat, sometimes showing one or several sharp maxima. Therefore, precisely identifying user-specific FOS seems essential before implementing an SSSEP-based BCI [5]. Additionally, we observed that the SSSEP's amplitude is positively correlated to the force and pressure of mechanical stimulation, up to a plateau of $0.05 \mathrm{~N}$ after which the amplitude of the SSSEP remains stable [43]. We also realised that the influence of the stimulation location on the SSSEP latency and spatial location has been scarcely studied. Further work in this direction seems desirable to gain a better understanding of SSSEPs. 


\section{SSSEP-based BCI: algorithms and performances}

In this section, we focus our review on SSSEP-based BCIs, describing their algorithms and performances, their similarities or at the opposite their originality compared to other approaches. We firstly discuss the main way to turn an SSSEP into a command usable in a BCI. Secondly, we present and comment on a synthetic table that summarises the methodologies, algorithms and performances of SSSEP-based BCIs. We finally present the most common algorithm described in the literature and compare it to less standard techniques found during our analysis of the state-of-the-art.

\subsection{Turning SSSEP into a usable BCI command}

In order to turn the SSSEP, or more precisely one of its characteristics, into a BCI command, it is necessary to specify which mental actions may influence it. In 2004, Giabbiconi et al observed that selective spatial attention towards the hand undergoing a sustained mechanical stimulation modulates the amplitude of the SSSEP elicited by the latter [15]. The authors stimulated the fingertips of the left and right index at 20 and $26 \mathrm{~Hz}$ respectively. Subjects were instructed "to attend to the flutter vibration at one finger while ignoring the other". An increase of the amplitude of the corresponding SSSEP results from this selective spatial attention, although the effect is highly subject dependent. This high between-subject variability motivated the author to use the standard error (SE), which is estimated by dividing the standard deviation by the square root of the number of samples. The authors reported an average increase of $30.6 \%$ ( $\mathrm{SE}=$ 11.2) of the amplitude of the SSSEP elicited in the right hemisphere when the attention was focused on the left index fingertips. Whereas when the attention was focused on the right index fingertips, the average augmentation of amplitude of the SSSEP elicited in the left hemisphere was $27.7 \%$ ( $\mathrm{SE}=11.0)$. The authors observed that the greatest SSSEP amplitude was measured at frontal electrode locations, contralateral to the stimulated index finger. The first article reporting on an SSSEP-based BCI, in 2006, used this attention focusing mental task [26].

\subsection{Synthesis of SSSEP-based BCIs: introduction}

We present and synthesize in tables $2 \mathrm{a}$ and $2 \mathrm{~b}$ the articles describing SSSEP-based BCIs obtained through our literature review. Table 2a presents the methodologies in the experiments, while table $2 \mathrm{~b}$ presents their main results. The two tables share the first 3 columns, used as an index: name of the first author, paper reference and stimulation location.
In table 2a, four columns present the methodology of each study: was a frequencies screening procedure involved? What task was performed by the subjects? How many subjects were tested? Which algorithms were used? We call "frequencies screening procedure" a user-specific procedure aiming to determine the resonance-like frequency, or at least the most efficient FOS, that will be later used in the BCI.

During the literature review, differences in the presentation of the task to the subject have been observed. We categorize the instruction provided to the subject into two categories: selective sensation (SS, stimulation-driven task) or attention focusing (AF, attention orientation-driven task). The instruction given in a SS task is correlated to the sensation produced by the stimulation. For example, the subject can be instructed to "select sensation on the indicated side of their hands as if stimulation on the attended side was stronger than on the unattended side" [48]. Whereas the instruction given in an AF task is focused on the shift of attention expected from the subject. The provided instruction can be: "focus attention on the finger stimulation indicated by the bar (cue)" [26]. We differentiate the instructions provided to the subject based on that difference between SS and AF tasks. Sometimes a small question mark (?) is inserted in table 2a, see column Task, when the authors did not provide enough information to differentiate the instruction type. In addition, some authors implemented a secondary task to help the subject concentrate on the main task. For example, in [26], the authors added a random transient amplitude twitch on the stimulation during some trials, that the subject had to detect and report to the experimenter.

We also sub-categorize the algorithm using three columns. Each column presents the answer to one of three different questions: how was the frequency information of the SSSEP used? How was the spatial information used? And how were the features extracted? Due to the limited amount of data collected during experiments, most of the studies used linear discriminant analysis (LDA) for classification. When the dimension of the extracted feature vector was relatively large, sometimes the "regularized" or "with shrinkage" version of LDA was used. This specific aspect will not be discussed here.

In table $2 \mathrm{~b}$, the main results of each study are presented. The classification accuracy (simply called "accuracy" thereafter) is reported, as well as its standard deviation when available. The $95 \%$ confidence limits of chance results for the considered classification problem is indicated in the column "chance level" when available [25]. Then a column indicates the type of classification problem, i.e. the number of classes that the algorithm was trained to 


\begin{tabular}{|c|c|c|c|c|c|c|c|c|}
\hline \multirow{2}{*}{ First author } & \multirow{2}{*}{ Ref. } & \multirow{2}{*}{$\begin{array}{c}\text { Stimulation } \\
\text { Location }\end{array}$} & \multirow{2}{*}{ Screen. } & \multirow{2}{*}{ Task } & \multirow{2}{*}{ Subj. } & \multicolumn{3}{|c|}{ Algorithm } \\
\hline & & & & & & Freq. & Spat. & Feat. \\
\hline Breitwieser & {$[7]$} & & Yes & $\mathrm{AF}$ & 14 & LAS & $\mathrm{CS}_{\mathrm{BM}}(1)$ & Amp. \\
\hline Ahn & {$[2]$} & & Yes & $\mathrm{MI} \mid \mathrm{AF}^{?}$ & 8 & $\mathrm{wBP}$ & CSP & $\mathrm{N} / \mathrm{A}$ \\
\hline Nam & {$[27]$} & & $\mathrm{No}^{1}$ & $\mathrm{SS}^{?}$ & 4 & $\begin{array}{c}\mathrm{wBP}+\mathrm{FFT} \\
\mathrm{wBP}+\mathrm{FFT} \\
(\mathrm{w}+\mathrm{n}) \mathrm{BP}+\mathrm{FFT}\end{array}$ & $\begin{array}{l}\text { None } \\
\text { CSP } \\
\text { CSP }\end{array}$ & $\begin{array}{l}\text { SPow. } \\
\text { SPow. } \\
\text { SPow. }\end{array}$ \\
\hline Ahn & {$[1]$} & & Yes & $\begin{array}{c}\text { MI \& SS } \\
\text { MI } \mid \text { SS }\end{array}$ & 16 & wBP & CSP & Pow? \\
\hline Severens & {$[39]$} & & $\mathrm{No}^{2}$ & $\mathrm{AF}^{?}$ & 12 & FFT & None & SPow. \\
\hline Müller-Putz & {$[26]$} & & Yes & $\mathrm{AF}$ & 5 & LAS & $\mathrm{CS}_{\mathrm{BM}}(2)$ & Log. Amp. \\
\hline Choi & {$[10]$} & & $\mathrm{No}^{3}$ & $\mathrm{AF}$ & 5 & $\mathrm{wBP}+\mathrm{FFT}$ & $\mathrm{CS}_{\mathrm{BM}}(2)$ & Rel. SPow. \\
\hline Breitwieser & {$[6]$} & & Yes & $\mathrm{AF}$ & 13 & $\mathrm{nBP}+\mathrm{LAS}$ & $\mathrm{CS}_{\mathrm{BM}}(13)$ & Log. Amp. \\
\hline Pokorny & {$[33]$} & & Yes & $\mathrm{AF}$ & 15 & $(\mathrm{w}+\mathrm{n}) \mathrm{BP}+\mathrm{LAS}$ & $\mathrm{CS}_{\mathrm{BM}}(13)$ & Log. Amp. \\
\hline Zhou & {$[49]$} & & $\mathrm{No}^{4}$ & MI & 13 & $\mathrm{wBP}+\mathrm{FFT}$ & CSP & SPow. \\
\hline Yao & {$[48]$} & & $\mathrm{No}^{5}$ & MI | SS & 11 & $\begin{array}{c}\mathrm{wBP} \\
\mathrm{wBP}+\mathrm{FFT}\end{array}$ & $\begin{array}{c}\text { CSP } \\
\text { CS (1) }\end{array}$ & $\begin{array}{c}\text { LV } \\
\text { SPow. }\end{array}$ \\
\hline Tao & {$[42]$} & & $\mathrm{No}^{4}$ & MI & 12 & $\mathrm{wBP}+\mathrm{FFT}$ & CS (1) & ISPC \\
\hline & & & & & & $\mathrm{wBP}+\mathrm{FFT}$ & CS (1) & SPow. cc ISPC \\
\hline Kim & {$[18]$} & & $\mathrm{No}^{6}$ & SS & 3 & $\begin{array}{l}\text { None } \\
\text { FFT }\end{array}$ & $\begin{array}{c}\text { CSP } \\
\text { CS (3), CSP }\end{array}$ & $\begin{array}{c}\text { LV } \\
\text { SPow. cc LV }\end{array}$ \\
\hline Kim & {$[19]$} & & Yes & SS & 4 & $\mathrm{wBP}+\mathrm{FFT}$ & CS (3), CSP & SPow. cc LV \\
\hline Kim & {$[20]$} & & Yes & SS & 5 & $\mathrm{wBP}+\mathrm{FFT}$ & CS (3), CSP & SPow. cc LV \\
\hline Kim & {$[21]$} & & & & & $\mathrm{wBP}+\mathrm{FFT}$ & CS (3) & SPow. \\
\hline Kim & {$[21]$} & & Yes & $\mathrm{MI} \mid \mathrm{SS}$ & 12 & $\begin{array}{c}\mathrm{wBP} \\
\mathrm{wBP}+\mathrm{FFT}\end{array}$ & CS (3), CSP & SPow. cc LV \\
\hline Kee & {$[17]$} & & Yes & $\mathrm{AF}$ & 5 & $\begin{array}{l}\text { wBD + None } \\
\text { wBD + PSD }\end{array}$ & $\begin{array}{l}\text { CSP } \\
\text { None }\end{array}$ & $\begin{array}{l}\text { LV } \\
\text { Pow. }\end{array}$ \\
\hline
\end{tabular}

Notes on screeningless SSSEP-based BCI, ${ }^{x}$ Material (size), Stimulation Shape:

$\begin{array}{lll}{ }^{1} \text { LRA }^{?}\left(0.79 \mathrm{~cm}^{2}\right), \text { Pulses } & { }^{3} \text { LRA }\left(2.5 \mathrm{~cm}^{2}\right) \text {, Vibration }(200 \mathrm{~Hz}) & { }^{5} \mathrm{C} 10-100 \mathrm{LRA}\left(0.79 \mathrm{~cm}^{2}\right), \text { Vibration }(175 \mathrm{~Hz}) \\ { }^{2} \text { Braille Stimulator, Pulses } & 4 \text { Electrical stimulation, Pulses } & { }^{6} \text { ERM, N/A }\end{array}$

Table 2a. EEG-based BCI exploiting SSSEP: Methods and Algorithms. All reported works use mechanical stimulation of the skin unless mentioned differently as an observation in table 2b. Legend and Acronyms: BM: bipolar montage; CS ( $x$ ): channel selection $x$ being the number of kept bipolar channel (if BM) monopolar channel otherwise; cc: concatenate; SPow.: power of selected frequencies (FOS) computed with FFT; Pow.: power computed with FFT; LAS: lock-in amplifier system; Amp.: amplitude output of a LAS; LV: log-variance of spatially filtered data from a CSP; ISPC: inter-stimulus phase coherence; nBP: narrow band-pass filter around stimulation frequencies $( \pm 1 \mathrm{~Hz})$; wBP: wide band-pass filters whose range can vary from $16-25 \mathrm{~Hz}[1]$ to $8-30 \mathrm{~Hz}[2]$; N/A: not available; *: irrespective of the paradigms or the algorithms; ?: unclear or ambiguous.

differentiate. Finally, a last column presents the main achievement of the study or provides supplementary information when needed.

\subsection{Signal processing and feature extraction}

In this section, we succinctly present the main techniques used in the reviewed articles in order to process the EEG signals and extract significant SSSEP features for further classification. Three categories of data processing will be considered: estimation of the frequency information contained in EEG signals, use or non-use of spatial information, type and characteristics of classification features.

4.3.1. Frequency information is retrieved from EEG signals with mostly three different approaches: Fourier transform, band-pass filtering or lock-in amplification.

The Fourier transform is the best known method for recovering the frequency information contained in a signal, through projections onto a base of sinusoidal components. Fourier-like transforms implemented in systems requiring real-time processing are usually 


\begin{tabular}{|c|c|c|c|c|c|c|}
\hline First author & Ref. & $\begin{array}{l}\text { Stimulation } \\
\text { Location }\end{array}$ & \begin{tabular}{|l} 
Accuracy \\
$(\mathrm{SSSEP})$
\end{tabular} & $\begin{array}{c}\text { Chance L. } \\
(p=5 \%)\end{array}$ & Problem & Observations \\
\hline Breitwieser & {$[7]$} & & $58.6 \% \pm 2.0$ & $61 \%$ & 2-class & \\
\hline Ahn & {$[2]$} & & $69 \% \pm 11$ & $\mathrm{~N} / \mathrm{A}$ & 2-class & $\begin{array}{l}\text { ERD from MI tasks reached an } \\
\text { average accuracy of } 71 \% \pm 10 .\end{array}$ \\
\hline Nam & {$[27]$} & & $\begin{array}{l}70.0 \\
57.1 \\
75.4\end{array}$ & $\mathrm{~N} / \mathrm{A}$ & 2-class & $\begin{array}{l}\text { Left thumb }(22 \mathrm{~Hz}) \text {, right thumb } \\
(27 \mathrm{~Hz})\end{array}$ \\
\hline Ahn & {$[1]$} & & $\approx 60 \%$ & $60 \%$ & 2-class & \\
\hline Severens & {$[39]$} & & $68 \%$ & $63.34 \%$ & 2-class & \\
\hline Müller-Putz & {$[26]$} & & $\begin{array}{l}\text { min: } 66.9 \% \\
\max : 83.9 \%\end{array}$ & $\mathrm{~N} / \mathrm{A}$ & 2-class & $\begin{array}{l}\text { Accuracy rather stable across days } \\
\text { (up to } 5 \text { days of experiments) }\end{array}$ \\
\hline Choi & {$[10]$} & & $65 \%$ & $\mathrm{~N} / \mathrm{A}$ & 2-class & Different patterns of stimulation \\
\hline Breitwieser & {$[6]$} & & $42.1 \% \pm 7.9$ & $39.8 \%$ & 3-class & Offline mean accuracy: $48.2 \% \pm 7.1$ \\
\hline Pokorny & {$[33]$} & & $48.6 \% \pm 6.2$ & $\begin{array}{c}40.8 \% \\
(p=1 \%)\end{array}$ & 3-class & $\begin{array}{l}9 \text { subjects above chance level, average } \\
\text { classification computed with them. }\end{array}$ \\
\hline Zhou & {$[49]$} & & $74.5 \% \pm 5.8^{\dagger}$ & $\mathrm{N} / \mathrm{A}$ & 2-class & $\begin{array}{l}\text { Classification of right hand MI vs. } \\
\text { idle based on the SSSEP's amplitude. } \\
\text { Uses electrical stimulations. }\end{array}$ \\
\hline Yao & {$[48]$} & & $72 \% \pm 15^{*}$ & $\mathrm{~N} / \mathrm{A}$ & 2-class & Left wrist $(23 \mathrm{~Hz})$, right wrist $(27 \mathrm{~Hz})$ \\
\hline Tao & {$[42]$} & & $\begin{array}{l}75.4 \% \pm 10.2 \\
75.7 \% \pm 13.9 \\
79.8 \% \pm 11.5\end{array}$ & $\mathrm{~N} / \mathrm{A}$ & 2-class & $\begin{array}{l}\text { Uses electrical stimulations and Inter- } \\
\text { Stimulus Phase Coherence (ISPC). }\end{array}$ \\
\hline Kim & {$[18]$} & & $\begin{array}{l}76 \% \\
80.93 \%\end{array}$ & $\mathrm{~N} / \mathrm{A}$ & 3-class & \\
\hline Kim & {$[19]$} & & $\mathrm{N} / \mathrm{A}$ & $\mathrm{N} / \mathrm{A}$ & 3-class & $\begin{array}{l}\text { Wheelchair-driving experiment. } \\
\text { Identical rhythm of commands be- } \\
\text { tween conditions: one every } 5 \mathrm{~s} \text {. } \\
\text { Goal reached in average in } 35 \mathrm{~s} \pm 2.1 \\
\text { (joystick) and } 101 \mathrm{~s} \pm 27.6 \text { (SSSEP). }\end{array}$ \\
\hline Kim & {$[20]$} & & $\mathrm{N} / \mathrm{A}$ & $\mathrm{N} / \mathrm{A}$ & 3 -class & Similar to [19]. \\
\hline Kim & {$[21]$} & & $\begin{array}{l}55 \% \pm 6.8^{\dagger} \\
73 \% \pm 11.8^{\dagger} \\
76 \% \pm 12.8^{\dagger}\end{array}$ & $\mathrm{N} / \mathrm{A}$ & 3-class & \\
\hline Kee & {$[17]$} & & $72 \% *$ & $\mathrm{~N} / \mathrm{A}$ & 2-class & \\
\hline
\end{tabular}

Table 2b. EEG-based BCI exploiting SSSEP: Performances. All of the reported works uses mechanical stimulation of the skin unless mentioned differently as an observation. Legend and Acronyms: N/A: not available; *: irrespective of the paradigms or the algorithms; ${ }^{\dagger}$ : extracted from a figure.

either the fast-Fourier transform (FFT) [21] or the power spectral density (PSD) estimation [17].

Unlike the other reviewed articles, Kee et al used the power information at all available frequencies in the FFT [17]. The most common approach though is to consider only some frequencies, e.g. the FOS and some of its harmonics [39]. The amplitudes or squared amplitudes of the sinusoidal components are then computed and provided as features to the classifier.

Another approach to the estimation of frequency information is narrow band-pass filtering. A narrow band-pass filter can be the first stage to recover the amplitude of a given frequency component of the EEG signal. In order to estimate the power of a signal inside a frequency band, it is common to square the band-pass filtered signal and average the result over time [22]. The authors of $[6,33]$ followed a slightly different 

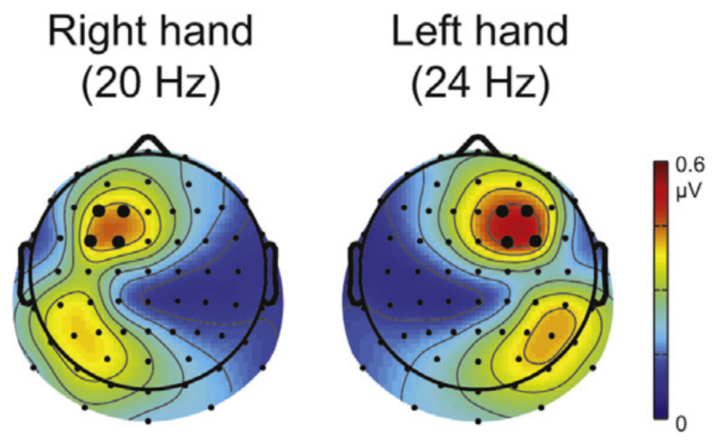

Figure 5. Grand average of SSSEP amplitudes, calculated across subjects and attention-related experimental condition (one-hand attended, one-hand ignored and both-hand attended). Figure reproduced with the appreciated authorisation of the authors from [31].

approach, combining a narrow band-pass filter with a lock-in amplifier, technique that will be presented in the next paragraph.

A lock-in amplifier or lock-in amplifier system (LAS) computes an estimate of the amplitude and phase of a specific sinusoidal component of a noisy signal. This method is fairly similar to a very narrow and tunable band-pass filter and can be considered as such [26]. In the literature, the amplitude estimation from the LAS is also averaged over a sliding time window of fixed length [26]. Commonly, the logarithm of the averaged amplitude is computed, for conditioning purposes and to obtain a distribution that approximates to normal [26]. Finally, either the averaged amplitude or its logarithm is fed into the classifier as a signal feature. This technique has long been known and frequently used when a single sinusoidal component is targeted in the EEG. Indeed, in a 1966 SSVEP study, Regan et al used the source of stimulation itself, modulated light, with an EEG to implement a physical LAS [35].

4.3.2. The spatial location of an SSSEP is well known and stable across subjects. Snyder showed that SSSEP can be principally recorded over the somatosensory cortex located in the post-central gyrus [40]. Moreover, the spatial location can be refined by building on the sensory homunculus that projects each part of the body onto a precise location of the sensory cortex. Therefore, a straightforward method to take advantage of this precise location is to process only the EEG signal recorded by a specific well-placed electrode. For example, the topomaps of figure 5 illustrate the positions of two concurrent SSSEP on the cortex.

For instance, to detect the SSSEP elicited by a mechanical stimulation of the right hand, one can process the signal recorded at location C3 of the 1020 international system of electrode placement. The spatial selection of signals of interest, in addition to the use of several FOS, allows SSSEP-based BCIs to provide independent and sometimes simultaneous control channels. This method is commonly used in the literature, we reported it as channel selection (CS) in table $2 \mathrm{a}$.

In order to reduce the noise, some authors use a bipolar montage (BM) $[26,10,33,6]$. The noise, or more generally a widespread and non task-related cortical activity, is removed by subtracting the signals recorded by two neighbour electrodes located next to the region of interest. For example, in an SSSEP-based $\mathrm{BCI}$, the signal corresponding to a noiseless virtual electrode over C3 is commonly obtained by subtracting the centro-parietal signal recorded at CP3 from the fronto-central signal recorded at FC3. It is worth noting also that the variance of the signal provided by the virtual electrode is lower than the sum of variances of both initial electrodes. More precisely, the variance of the BM signal is the sum of variances of the initial signals minus twice their covariance, the latter being usually high since the electrodes are close to each other. Electrodes with a lower variance are assumed to have a better signal-to-noise ratio. This property actually derives from the principle of "blocking" well known in statistics.

We also noticed that the common spatial pattern (CSP) filtering method was regularly used. CSP is the gold standard spatial filtering technique in motorimagery-based BCIs (MI) [22]. This algorithm was indeed part of the methodology in all studies that compare SSSEP-based BCIs to MI-based BCI [1, 48, 21]. The CSP algorithm determines spatial filters, i.e. weights of a linear combination of EEG signals, that maximize the variance of the resulting signal for one class while minimizing it for the other classes. Here, one class corresponds to the set of signals containing SSSEPs elicited by a specific stimulation, and the other class to the set of all other signals available in the recorded dataset. Typically, most of the studies use 3 pairs of spatial filters computed by the CSP algorithm, i.e. 3 filters that maximize the variance of one class and 3 that maximize the variance of the other class. The logarithms of variance of the CSP filtered EEG signals provide the feature vector fed into the classifier, with dimension 6 in the previous example.

\subsection{Synthesis of SSSEP-based BCIs: main results}

In all the studies of tables $2 \mathrm{a}$ and $2 \mathrm{~b}$ except one, different limbs were selected for receiving each periodical somatosensory stimulus when more than one was considered. However, in [7], Breitwieser et al implemented a BCI using two fingers of the same hand. The reported average discrimination accuracy between both fingers, across all subjects, was $58.6 \% \pm 2.0$. This 
result is below chance level (61\%) considering the number of trials and a confidence limit at 95\% [25]. However, the attention focusing effect, for one finger vs. resting state, both during stimulation, was successfully highlighted. The accuracy related to AF was similar for both fingers, i.e. thumb or middle finger, at about $66.5 \% \pm 5.4$.

Usually, only one of the two stimulation techniques, i.e. short mechanical pulses or vibro-tactile stimulation, is used in the presented studies. In [10], Choi et al tested different techniques of stimulation. The accuracy reported in table $2 \mathrm{~b}$ for this article is the one for the control condition used in their experiment, i.e. for a standard vibro-tactile stimulation. Other compared techniques were variations of this vibro-tactile stimulation, with a carrier frequency at $200 \mathrm{~Hz}$ amplitude-modulated by non-periodic or periodic signals. One of the tested models was named "random pulses", in which, during the standard vibrotactile stimulation, transient random decreases of stimulation amplitude were inserted. Another tested model was named "tic-tic-toc", in which during the standard vibro-tactile stimulation, a rhythmic train of transient decreases of stimulation amplitude was added. Two out of three pulses were regular pulses (tic) and the other one was much stronger (toc). Once the "toc" was added, the pattern restarted from the first "tic", during the whole stimulation. This stimulation technique yields a significant improvement of the accuracy ( $\mathrm{p}<0.0001$, post-hoc Tukey tests) compared to the others. The size effect was not reported, but we may deduce from the experiment description that the median accuracy of the tic-tic-toc pattern was around $75 \%$, while the median accuracies of standard vibro-tactile and random pulses were respectively $65 \%$ and $70 \%$.

Most of the authors assessed the accuracy of their SSSEP-based BCI in highly controlled conditions. Usually, subjects were seated in front of a screen and performed the same task repetitively. Kim et al followed a different approach [19, 20, 21]. Their evaluation framework consisted in driving a wheelchair along a predefined course while avoiding obstacles. The rate of wheelchair commands, one every five seconds, was the same for the tested SSSEP-based $\mathrm{BCI}$ and the control condition, i.e. a joystick. Three mechanical stimuli were simultaneously provided to the subjects at their right and left index fingertip and at the big toe of the right foot. The subject emitted a command by "concentrating his attention on the vibration stimulus", task referred to as SS in table $2 \mathrm{a}$. The ending point of the predefined path followed by the wheelchair was roughly $10 \mathrm{~m}$ apart from the starting point. Similar performances were obtained within the three studies, the ending point being reached on average twice faster with a joystick than with the BCI.
In tables $2 \mathrm{a}$ and $2 \mathrm{~b}$, most of the studies involved mechanical stimulation of fingers or, to a lesser extent, wrists. Foot-elicited SSSEPs have been scarcely studied, but we have found an example in the work of Kee et al [17]. The authors asked the subjects to "attend their corresponding feet" when a cue was provided, task referred to as AF in table 1a. This AF task allowed the subjects to discriminate between both feet with an average accuracy of $72.6 \%$.

4.4.1. Performance stability of SSSEP-based BCIs. We already indicated in section 3 that user-specific SSSEP characteristics seem to remain stable within a few weeks in between experiments [5]. Here, we aim to provide information about the stability over time of accuracy in SSSEP-based BCIs.

We found only one study that evaluates specifically the accuracy of an SSSEP-based BCI over several days. In 2006, Müller-Putz et al reported an accuracy ranging from 66.9 to $83.9 \%$ across subjects on day one of the experiment [26]. Among the five subjects, three performed 4 successive sessions (one per day), and one performed 5 sessions. For each subject, online and offline accuracies were found rather stable from one session to another, except for subject 5 who performed 4 sessions and for whom an increase was observed in online as well as offline accuracies. For this subject, online accuracy increased from $63.8 \%$ (day 2 ) to $71.7 \%$ (day 4) whereas offline accuracy increased from $64.4 \%$ (day 1) to $75.0 \%$ (day 4 ).

4.4.2. Frequency-based detection versus spatialbased detection is, in the reviewed literature, the mainstream comparison between two algorithms that has been reported.

Kee et al compared the accuracy obtained when using different features, extracted by a CSP or a PSD, in a 2-class classification problem [17]. First of all, in both methods, each EEG signal was filtered by a fourth order Butterworth wide band-pass filter between 0.5 and $40 \mathrm{~Hz}$. This band-pass was introduced to capture specifically the alpha and beta frequency bands. After a frequencies screening procedure aiming to assess the resonance-like frequency for each of their subjects, the authors used subject-specific FOS. The results from the frequencies screening procedure shows a broad distribution of resonance-like frequency, across subjects, the resonance-like frequency set was composed of the following FOS: 13, 23, 25, and 29. A PSD estimation computed on EEG epochs, lasting 250 milliseconds and sampled at $125 \mathrm{~Hz}$, yielded a feature vector grouping the powers at 10 frequencies between 4 and $40 \mathrm{~Hz}$.

Thus, although they determined the resonancelike frequency for each subject, the authors did not 
use it for tuning the parameters of signal processing. Consequently, because of the lack of selectivity, one can wonder if the proposed technique does not detect in fact a fluctuation within the alpha or beta band resulting from the AF task, instead of a specific variation of the SSSEP amplitude induced by AF. For their second method, the authors used the $\log$ variance of EEG signals filtered with 3 CSP pairs as classification features. They report similar accuracies for both algorithms, respectively $72.6 \%$ for PSD and $72.2 \%$ for CSP.

In 2014, Kim et al compared the accuracies of an SSSEP-based BCI for different extracted features [18]. The first tested feature vector groups the log variances of 6 CSP filtered signals. The second tested feature vector gathers CSP-based log variances and several power measurements at specific frequencies determined by a FFT. The power estimation was at least determined at the FOS, but the procedure as well as the parameters of the FFT (window size, sampling frequency) were not reported. Surprisingly, the authors did not compute the FFT on spatially filtered signals, but on raw EEG signals. Performance evaluation was not performed either for feature vectors containing only the powers estimated by FFT. The CSP-based method yields an average accuracy of $76 \%$, whereas the CSP + FFT-based method yields an average accuracy of $80.93 \%$. The experiment was performed on only three subjects, which impairs the statistical significance of results.

In 2018, Kim et al performed an equivalent study, this time on a total of twelve subjects [21]. The accuracy obtained with feature vectors containing only the powers estimated by a FFT was also computed. The CSP and FFT-based methods yield average accuracies of $73 \% \pm 11.8$ and $55 \% \pm 6.8$ respectively. The joint feature vector, including both CSP and FFT attributes, yields an average accuracy of $76 \% \pm 12.8$.

It is noteworthy that, to a lesser extend, one publication also uses phrase information [42]. The Inter-Stimulus Phase Coherence is computed using the Hilbert transform and the analytic signal form of the EEG. This signal processing technique increases the accuracy of the proposed system from $75.4 \%$ to $79.8 \%$.

4.4.3. Comparison and combination of SSSEP-based $B C I s$ with MI-based BCIs. In tables 2a and 2b, several studies compared the accuracies of an SSSEPbased BCI and a MI-based BCI, such as [2, 48, 1, 21].

In 2014, Yao et al conducted a 4-class experiment (left/right MI and left/right SS). The authors retained 6 combinations of conditions for a 2-class classification task by extracting two different groups of data, for example, right-SS vs. left-MI [48]. Four out of the 6 combinations were referred to as hybrid, such as in the previously provided example, when the two considered groups did not correspond to the same modality. The accuracy reported in table $2 \mathrm{~b}$ is for the non-hybrid combination with only SS. This combination yield an accuracy of $72.6 \% \pm 14.8$, which is comparable to the accuracies of other studies.

For most subjects, using hybrid modalities had a limited impact on the accuracy. However, for two subjects, using hybrid modalities yielded an accuracy increase from $55-60 \%$ and $70 \%$ to above $90 \%$. It is noticeable that the authors used fixed $23 \mathrm{~Hz}$ (left wrist) and $27 \mathrm{~Hz}$ (right wrist) FOS and a fixed wide band-pass filter between 8 and $26 \mathrm{~Hz}$, that does not include the right FOS. Feature extraction was performed using 3 pairs of spatial filters computed with a CSP, the log variance of the filtered signals was used. Therefore, we can wonder if this analysis framework does not take mostly into account the spatial information of the left wrist-elicited SSSEP mixed with the influence of attention focusing. Among all combinations, the left SS vs. right MI produces the best performance with an average accuracy of $83.1 \% \pm 10.4$.

In 2014, Ahn et al also compared and combined MI and SSSEP-based BCI paradigms [1]. The authors assessed the accuracies for different conditions: MI alone, SS alone, and both mental tasks at the same time. The subject received the same mechanical stimulation during these three conditions. A fourth condition was tested, in which the subject performed a SS task followed by a MI task, but without stimulation. The three conditions with stimulation lead to similar accuracies around $60 \%$ while MI without stimulation reached $70 \%$. Actually, this methodology was very similar to the one of Yao et al [48], except that the band-pass filter was different for each condition. The wide band-pass filter was between 16 to $25 \mathrm{~Hz}$ for the SS condition and 8 to $15 \mathrm{~Hz}$ for the MI without stimulation condition. Every resonance-like frequency identified during the frequencies screening procedure lies within 16-25 Hz. For each condition, feature extraction was performed using CSP spatial filters, 5 pairs of filters. The vector formed from the power of the filtered signals was fed into the classifier.

In 2018, Kim et al also compared both paradigms [21]. They evaluated an SSSEP-based BCI as well as a MI-based BCI for a wheelchair-driving task. The authors reported that the MI-based BCI was less efficient than the SSSEP-based BCI, with an average path following duration of $173 \pm 95$ seconds for MI compared to $102 \pm 26$ seconds for SSSEP. One can notice that with the MI-based BCI, four subjects could not finish the path following task in less than 5 minutes and that in this case a 300 seconds duration of the task was considered for calculating average performance. When these subjects are removed from the list 
to estimate the average task duration, the latter drops down to $110 \pm 26$ seconds, which is equivalent to the average duration of the SSSEP-based BCI task.

4.4.4. Comparison of SSSEP-based BCI with a somatosensory-evoked P300-based BCI was very rarely performed in the literature. We report only one study that performed this comparison.

In 2013, Severens et al compared their SSSEPbased BCI with a P300-based BCI in which the P300 was elicited by a transient mechanical stimulation [39]. The SSSEP was elicited by short mechanical pulses, with a $50 \%$ duty-cycle control signal. The authors reported that the P300 alone outperformed the SSSEPbased BCI, with an average accuracy of $74 \%$ and $60 \%$ respectively, after only 2 seconds of recording. With 16 seconds of recording, the respective accuracies increased to $93 \%$ and $68 \%$. The SSSEP-based BCI reached this level of accuracy after 4 seconds, and remains stable after that, whereas the P300-based BCI accuracy kept increasing with recording duration. Additionally, the accuracy of the combination of both stimulation, i.e. transient twitches and sustained pulsation, does not significantly differ from the one of P300 alone.

However, the differences of performance can be put in perspective from a different point of view. Firstly, the features chosen for SSSEP classification are unusual, since they correspond to the power estimates of EEG signals at the two FOS, 18 and $21 \mathrm{~Hz}$, at two of its harmonics as well as at half of the FOS. EEG was recorded with 64 electrodes, among which the authors performed an outlier detection for each subject, resulting in removing an average of 6.9 electrodes (std 3.8). Anyway, this yields a very large feature vector, with 8 signal powers (at $9,10.5,18,21,36,42,54$, and $63 \mathrm{~Hz}$ ) for each retained electrode. As seen in section 3.4.2, other studies tend to demonstrate that powers at harmonics contain little information, especially those produced by FOS higher than $11 \mathrm{~Hz}$. Moreover, when the stimulation results from short mechanical pulses, i.e. from a square signal, there is no information at the second harmonic of the FOS. Therefore, since no prior feature selection is performed before classification, its accuracy can be seriously impacted. This effect may have been counterbalanced by the use of a regularized classification algorithm, but this has not been reported or discussed by the authors.

To summarise, in this section we presented a review of studies describing an EEG-based BCI exploiting SSSEP. We firstly presented the main signal processing and feature extraction techniques reported in the literature. In most studies, features are extracted by a FFT analysis, or by an equivalent technique. Lock-in amplifier systems are also regularly used. Spatial filtering techniques, such as common spatial pattern or channel selection with bipolar montage, can improve SSSEP analysis.

The second part of our review was focused on analysing the performances of the EEG-based BCI exploiting SSSEP. Reported average accuracies, for systems exploiting the stimulation of two distinct upper limbs, varies from $60 \%$ to $79.8 \%$, for a 2 class problem. 3-class problems are rarely tackled, and BCI performance shows higher variability, with an accuracy from $42.1 \%$ to $80.93 \%$. One study uses SSSEP to reduce the number of false triggering in MI-based BCI [49]. Comparison of SSSEP-based BCI with paradigms other than motor imagery have been scarcely performed, which reinforces our idea that the field should be further explored.

\section{Conclusion}

In this review, we presented and analysed 24 articles that deal either with the estimation of user specific characteristics of SSSEPs or with the description of an SSSEP-based BCI.

Most studies describing the precise relationship between SSSEP characteristics and stimulation parameters, show that they are highly subject dependant. Due to this strong variability, the frequencies screening procedure, i.e. the determination of user-specific FOS, appears as a mandatory stage in any SSSEP-based BCI in order to improve its performance. For instance, the study of Pokorny et al shows that a shift of the FOS by $5 \mathrm{~Hz}$ from the resonance-like frequency elicits SSSEPs with an amplitude roughly twice weaker [33]. However, although they are of major interest, the results of the determination of user-specific FOS or the procedure itself are rarely reported in their entirety as can be seen in table $2 \mathrm{~b}$.

We also presented 17 studies that describe an SSSEP-based BCI and assess its performance. The reported average accuracy for a 2-class classification problem varies between $60 \%$ to $72 \%$, and between $42.1 \%$ to $76 \%$ for a 3-class classification problem. However, in most of the studies reporting high average accuracies, experiments were performed with a small number of subjects, which lowers their statistical significance.

Some of the presented studies compare their results to other BCI paradigms. They provide very interesting insights, but again due to the small number of subjects involved in the experiments, no definite conclusion can be drawn. Paradigm comparison was mostly performed between MI and SSSEP. SSSEP has the advantage of being easier to detect in the EEG, thanks to the use of exogenous stimuli, than MI-related 
cortical activities. False command triggering, caused by the detection of irrelevant mental activity during MI, can also be mitigated by the use of SSSEP [49].

As we stated in the introduction, SSSEP-based BCI uses a modality that is underused or unusable by a person with a motor disability. Hence, SSSEPbased BCIs, that do not monopolize the user's sight or hearing for stimulation or feedback, should be developed and tested for motor-disabled users. All the experiments reported in the reviewed articles were performed on healthy people. However, it has been shown that people who suffer from amyotrophic lateral sclerosis of from other neuromuscular pathologies such as spinal muscular atrophy retain a functional somatosensory system $[14,16]$. It offers the hope that future developments of SSSEP-based BCIs will provide these patients with an easy to use interface that does not solicit their visual or auditory system.

After this review, we can also conclude that studies show that SSSEPs characteristics highly depend on the stimulation procedure or device. The use of a standard and validated stimulation unit, conforming with safety standards for medical electrical equipment such as EN 60601-1:2006 [32], might reduce this variability and allow an easier comparison of results from different studies.

Finally, we also lack answers to many questions about SSSEP that initially look rather basic, such as: What is the influence of the stimulation position on the characteristics of the SSSEP, like amplitude or total latency? How long does it take for a SEP to reach its steady-state? Is there a relationship between physical traits of a person and resonance-like frequencies? This research field should be investigated further to gain an in-depth understanding of SSSEPs and pave the way to efficient SSSEP-based BCIs.

\section{Bibliography}

[1] Aнn, S. ; Aнn, M. ; Сно, Н. ; Jun, S. C.: Achieving a hybrid brain-computer interface with tactile selective attention and motor imagery. In: Journal of Neural Engineering 11 (2014), Nr. 6, 066004. http:// dx.doi.org/10.1088/1741-2560/11/6/066004. - DOI 10.1088/1741-2560/11/6/066004. - ISSN 1741-2552

[2] Aнn, S. ; Jun, S. C.: Feasibility of hybrid BCI using ERD- and SSSEP- BCI. In: 2012 12th International Conference on Control, Automation and Systems, 2012, S. $2053-2056$

[3] Ahn, S. ; KIM, K. ; Jun, S. C.: Steady-State Somatosensory Evoked Potential for Brain-Computer Interface-Present and Future. In: Frontiers in Human Neuroscience 9 (2016), 716. http://dx.doi.org/10.3389/fnhum. 2015 . 00716. - DOI 10.3389/fnhum.2015.00716. - ISSN 16625161

[4] Allison, B. Z. ; Kübler, A. ; Jin, J.: 30+ years of P300 brain-computer interfaces. In: Psychophysiology 57 (2020), Nr. 7. http://dx.doi.org/10.1111/psyp. 13569. - DOI 10.1111/psyp.13569. - ISSN 0048-5772, 14698986
[5] Breitwieser, C. ; Kaiser, V. ; Neuper, C. ; MüllerPutz, G. R.: Stability and distribution of steadystate somatosensory evoked potentials elicited by vibrotactile stimulation. In: Medical \& Biological Engineering 83 Computing 50 (2012), Nr. 4, 347-357. http: //dx.doi.org/10.1007/s11517-012-0877-9. - DOI 10.1007/s11517-012-0877-9. - ISSN 1741-0444

[6] Breitwieser, C. ; Pokorny, C. ; Müller-Putz, G. R.: A hybrid three-class brain-computer interface system utilizing SSSEPs and transient ERPs. In: Journal of Neural Engineering 13 (2016), Nr. 6, 066015. http: //dx.doi.org/10.1088/1741-2560/13/6/066015. - DOI 10.1088/1741-2560/13/6/066015

[7] Breitwieser, C. ; Pokorny, C. ; Neuper, C. ; MüllerPutz, G. R.: Somatosensory evoked potentials elicited by stimulating two fingers from one hand - Usable for BCI? In: 2011 Annual International Conference of the IEEE Engineering in Medicine and Biology Society, 2011, S. 6373-6376

[8] Brickwedde, M. ; Schmidt, M. D. ; Krüger, M. C. ; Dinse, H. R.: $20 \mathrm{~Hz}$ Steady-State Response in Somatosensory Cortex During Induction of Tactile Perceptual Learning Through LTP-Like Sensory Stimulation. In: Frontiers in Human Neuroscience 14 (2020), S. 257. http://dx.doi.org/10.3389/fnhum.2020.00257. - DOI 10.3389/fnhum.2020.00257. - ISSN 1662-5161

[9] Cheveigné, A. de ; Nelken, I.: Filters: When, Why, and How (Not) to Use Them. In: Neuron 102 (2019), Nr. 2, 280-293. http://dx.doi.org/10.1016/j.neuron. 2019. 02.039. - DOI 10.1016/j.neuron.2019.02.039. - ISSN 0896-6273

[10] Choi, I. ; Bond, K. ; Krusienski, D. ; Nam, C. S.: Comparison of Stimulation Patterns to Elicit SteadyState Somatosensory Evoked Potentials (SSSEPs): Implications for Hybrid and SSSEP-Based BCIs. In: 2015 IEEE International Conference on Systems, Man, and Cybernetics, 2015, S. 3122-3127

[11] Colon, E. ; Legrain, V. ; Mouraux, A.: Steady-state evoked potentials to study the processing of tactile and nociceptive somatosensory input in the human brain. In: Clinical Neurophysiology 42 (2012), Nr. 5, S. 315323. http://dx.doi.org/10.1016/j.neucli.2012.05. 005. - DOI 10.1016/j.neucli.2012.05.005. - ISSN 17697131

[12] Erp, J. B. F. v. ; Brouwer, A.: Touch-based Brain Computer Interfaces: State of the art. In: 2014 IEEE Haptics Symposium (HAPTICS), 2014, S. 397-401

[13] Fleury, M. ; Lioi, G. ; Barillot, C. ; Lécuyer, A.: A Survey on the Use of Haptic Feedback for BrainComputer Interfaces and Neurofeedback. In: Frontiers in Neuroscience 1 (2020). http://dx.doi.org/10.3389/ fnins.2020.00528. - DOI 10.3389/fnins.2020.00528

[14] Georgesco, M. ; Salerno, A. ; Camu, W.: Somatosensory evoked potentials elicited by stimulation of lower-limb nerves in amyotrophic lateral sclerosis. In: Electroencephalography and Clinical Neurophysiology/Evoked Potentials Section 104 (1997), Nr. 4, 333-342. http: //dx.doi.org/10.1016/S0168-5597(97)00018-X. - DOI 10.1016/S0168-5597(97)00018-X. - ISSN 0168-5597

[15] Giabbiconi, C. M. ; Dancer, C. ; Zopf, R. ; Gruber, T. ; Müller, M. M.: Selective spatial attention to left or right hand flutter sensation modulates the steadystate somatosensory evoked potential. In: Cognitive Brain Research 20 (2004), Nr. 1, 58-66. http://dx. doi.org/10.1016/j.cogbrainres.2004.01.004. - DOI 10.1016/j.cogbrainres.2004.01.004. - ISSN 0926-6410

[16] Hamada, M. ; Hanajima, R. ; Terao, Y. ; Sato, F. ; Okano, T. ; Yuasa, K. ; Furubayashi, T. ; Okabe, S. ; Arai, N. ; Ugawa, Y.: Median nerve somatosensory evoked potentials and their high- 
frequency oscillations in amyotrophic lateral sclerosis. In: Clinical Neurophysiology 118 (2007), Nr. 4, 877886. http://dx.doi.org/10.1016/j.clinph.2006.12. 001. - DOI 10.1016/j.clinph.2006.12.001. - ISSN 13882457

[17] Kee, Y. ; Won, D. ; Lee, S.: Classification of left and right foot movement intention based on steadystate somatosensory evoked potentials. In: 2017 th International Winter Conference on Brain-Computer Interface (BCI), IEEE, 2017. - ISBN 978-1-5090-50963, 106-108

[18] Kim, K. ; LeE, S.: Steady-state somatosensory evoked potentials for brain-controlled wheelchair. In: 2014 International Winter Workshop on Brain-Computer Interface (BCI), IEEE, 2014. - ISBN 978-1-4799-2588$9,1-2$

[19] Kim, K. ; LeE, S.: Wheelchair Control Based on SteadyState Somatosensory Evoked Potentials. In: 2015 IEEE International Conference on Systems, Man, and Cybernetics, 2015, S. 1504-1507

[20] KIM, K. ; LEE, S.: Towards an EEG-based intelligent wheelchair driving system with vibro-tactile stimuli. In: 2016 IEEE International Conference on Systems, Man, and Cybernetics (SMC), 2016, S. 002382-002385

[21] Kim, K. ; Suk, H. ; LeE, S.: Commanding a BrainControlled Wheelchair Using Steady-State Somatosensory Evoked Potentials. In: IEEE transactions on neural systems and rehabilitation engineering: a publication of the IEEE Engineering in Medicine and Biology Society 26 (2018), Nr. 3, S. 654-665. http: //dx.doi.org/10.1109/TNSRE. 2016.2597854. - DOI 10.1109/TNSRE.2016.2597854. - ISSN 1534-4320

[22] Lotte, F. ; Bougrain, L. ; Clerd, M.: Electroencephalography (EEG)-Based Brain-Computer Interfaces. Version: 2015. http://dx.doi.org/10.1002/ 047134608X. W8278. In: WeBster, J. G. (Hrsg.): Wiley Encyclopedia of Electrical and Electronics Engineering. John Wiley \& Sons, Inc., 2015. - DOI 10.1002/047134608X.W8278. - ISBN 978-0-471-34608$1,1-20$

[23] Moher, D. ; Liberati, A. ; Tetzlaff, J. ; Altman, D. G.: Preferred reporting items for systematic reviews and meta-analyses: the PRISMA statement. In: PLoS Medicine 6 (2009), Nr. 7, 1-6. http://dx.doi.org/10. 1136/bmj.b2535. - DOI 10.1136/bmj.b2535. - ISSN $1756-1833$

[24] Müller-Putz, G. R. ; Neuper, C. ; Pfurtscheller, G.: "Resonance-like" Frequencies of Sensorimotor Areas Evoked by Repetitive Tactile Stimulation - Resonanzeffekte in sensomotorischen Arealen, evoziert durch rhythmische taktile Stimulation. In: Biomedizinische Technik - BIOMED TECH 46 (2001), Nr. 7-8, S. 186-190. http: //dx.doi.org/10.1515/bmte.2001.46.7-8.186. - DOI 10.1515/bmte.2001.46.7-8.186

[25] Müller-Putz, G. R. ; Scherer, R. ; Brunner, C. ; Leeb, R. ; Pfurtscheller, G.: Better than random? A closer look on BCI results. In: International journal of bioelectromagnetism 10 (2008), Nr. 1, 52-55. https://graz.pure.elsevier.com/en/publications/ better-than-random-a-closer-look-on-bci-results

[26] Müller-Putz, G. R. ; Scherer, R. ; Neuper, C. ; Pfurtscheller, G.: Steady-state somatosensory evoked potentials: suitable brain signals for braincomputer interfaces? In: IEEE Transactions on Neural Systems and Rehabilitation Engineering 14 (2006), Nr. 1, S. 30-37. http://dx.doi.org/10.1109/TNSRE. 2005. 863842. - DOI 10.1109/TNSRE.2005.863842

[27] Nam, Y. ; Cichocki, A. ; ChOI, S.: Common spatial patterns for steady-state somatosensory evoked potentials. In: 35th Annual International Confer- ence of the IEEE Engineering in Medicine and Biology Society (EMBC) (2013), S. 2255-2258. http: //dx.doi.org/10.1109/EMBC.2013.6609986. - DOI 10.1109/EMBC.2013.6609986

[28] Namerow, N. S. ; Sclabassi, R. J. ; Enns, N. F.: Somatosensory responses to stimulus trains: normative data. In: Electroencephalography and Clinical Neurophysiology 37 (1974), Nr. 1, S. 11-21. http: //dx.doi.org/10.1016/0013-4694(74)90241-7. - DOI 10.1016/0013-4694(74)90241-7. - ISSN 0013-4694

[29] Noss, R. S. ; Boles, C. D. ; Yingling, C. D.: Steadystate analysis of somatosensory evoked potentials. In: Electroencephalography and Clinical Neurophysiology 100 (1996), Nr. 5, S. 453-461. http://dx.doi.org/ 10.1016/0168-5597(96)96011-6. - DOI 10.1016/01685597(96)96011-6. - ISSN 0013-4694

[30] Nuwer, M. R. ; Dawson, E. G. ; Carlson, L. G. ; Kanim, L. E. ; Sherman, J. E.: Somatosensory evoked potential spinal cord monitoring reduces neurologic deficits after scoliosis surgery: results of a large multicenter survey. In: Electroencephalography and clinical neurophysiology 96 (1995), Nr. 1, S. 6-11. http://dx.doi.org/10. 1016/0013-4694(94)00235-D. - DOI 10.1016/00134694(94)00235-D

[31] Pang, C. Y. ; Mueller, M. M.: Test-retest reliability of concurrently recorded steady-state and somatosensory evoked potentials in somatosensory sustained spatial attention. In: Biological Psychology 100 (2014), Juli, S. 86-96. http://dx.doi.org/10.1016/j.biopsycho. 2014.05.009. - DOI 10.1016/j.biopsycho.2014.05.009. ISSN $1873-6246$

[32] Pokorny, C. ; Breitwieser, C. ; Müller-Putz, G. R.: A Tactile Stimulation Device for EEG Measurements in Clinical Use. In: IEEE Transactions on Biomedical Circuits and Systems 8 (2014), Nr. 3, S. 305-312. http: //dx.doi.org/10.1109/TBCAS.2013.2270176. - DOI 10.1109/TBCAS.2013.2270176

[33] Pokorny, C. ; Breitwieser, C. ; Müller-Putz, G. R.: The Role of Transient Target Stimuli in a Steady-State Somatosensory Evoked Potential-Based Brain-Computer Interface Setup. In: Frontiers in Neuroscience 10 (2016), S. 152. http://dx.doi.org/10.3389/fnins . 2016.00152. - DOI 10.3389/fnins.2016.00152. - ISSN $1662-4548$

[34] Punsawad, Y.; Wongsawat, Y.: Multi-command SSAEPbased BCI system with training sessions for SSVEP during an eye fatigue state. In: IEEJ Transactions on Electrical and Electronic Engineering 12 (2017), S. s72s78. http://dx.doi.org/10.1002/tee.22441. - DOI $10.1002 /$ tee. 22441

[35] Regan, D.: Some characteristics of average steady-state and transient responses evoked by modulated light. In: Electroencephalography and Clinical Neurophysiology 20 (1966), Nr. 3, 238-248. http://dx.doi.org/10. 1016/0013-4694(66)90088-5. - DOI 10.1016/00134694(66)90088-5. - ISSN 00134694

[36] Regan, D.: Comparison of Transient and Steady-State Methods. In: Annals of the New York Academy of Sciences 388 (1982), Nr. 1, S. 45-71. http://dx. doi.org/10.1111/j.1749-6632.1982.tb50784.x. - DOI 10.1111/j.1749-6632.1982.tb50784.x. - ISSN 1749-6632

[37] REgAN, D.: Human brain electrophysiology: evoked potentials and evoked magnetic fields in science and medicine. Elsevier, 1989. - ISBN 978-0-444-01324-8. - Open Library ID: OL2044400M

[38] Schmidt, R. F. (Hrsg.): Fundamentals of Sensory Physiology. Springer-Verlag, 1986. http://dx.doi.org/ 10.1007/978-3-662-01128-7. http://dx.doi.org/10. 1007/978-3-662-01128-7. - ISBN 978-3-642-82598-9

[39] Severens, M. ; Farquhar, J. ; Duysens, J. ; Desain, 
P.: A multi-signature brain-computer interface: Use of transient and steady-state responses. In: Journal of neural engineering 10 (2013), Nr. 2, S. 026005. http: //dx.doi.org/10.1088/1741-2560/10/2/026005. - DOI 10.1088/1741-2560/10/2/026005

[40] SNyder, A. Z.: Steady-state vibration evoked potentials: descriptions of technique and characterization of responses. In: Electroencephalography and Clinical Neurophysiology 84 (1992), Nr. 3, S. 257-268. http: //dx.doi.org/10.1016/0168-5597(92)90007-x. - DOI 10.1016/0168-5597(92)90007-x. - ISSN 0013-4694

[41] Su, S. ; Chai, G. ; Shu, X. ; Sheng, X. ; Zhu, X.: Electrical stimulation-induced SSSEP as an objective index to evaluate the difference of tactile acuity between the left and right hand. In: Journal of Neural Engineering 17 (2020), Nr. 1, S. 016053. http://dx.doi.org/10.1088/ 1741-2552/ab5ee9. - DOI 10.1088/1741-2552/ab5ee9. - ISSN 1741-2552

[42] TAO, X. ; YI, W. ; WANG, K. ; He, F. ; QI, H.: Interstimulus phase coherence in steady-state somatosensory evoked potentials and its application in improving the performance of single-channel MI-BCI. In: Journal of Neural Engineering 18 (2021), Nr. 4. http://dx.doi. org/10.1088/1741-2552/ac0767. - DOI 10.1088/17412552/ac0767. - ISSN 1741-2552

[43] Tobimatsu, S. ; Zhang, Y. M. ; Kato, M.: Steadystate vibration somatosensory evoked potentials: physiological characteristics and tuning function. In: Clinical Neurophysiology: Official Journal of the International Federation of Clinical Neurophysiology 110 (1999), Nr. 11, 1953-1958. http://dx.doi.org/10. 1016/S1388-2457(99)00146-7. - DOI 10.1016/S13882457(99)00146-7. - ISSN 1388-2457

[44] Tobimatsu, S. ; Zhang, Y. M. ; Suga, R. ; Kato, M.: Differential temporal coding of the vibratory sense in the hand and foot in man. In: Clinical Neurophysiology 111 (2000), Nr. 3, 398-404. http://dx.doi.org/10. 1016/S1388-2457(99)00278-3. - DOI 10.1016/S13882457(99)00278-3. - ISSN 1388-2457

[45] Toleikis, J. R.: Intraoperative Monitoring Using Somatosensory Evoked Potentials. In: Journal of Clinical Monitoring and Computing 19 (2005), Nr. 3, 241258. http://dx.doi.org/10.1007/s10877-005-4397-0. - DOI 10.1007/s10877-005-4397-0. - ISSN 1573-2614

[46] Wang, H. ; Ge, Y. ; Song, A. ; LI, B. ; XU, B.: The vibro-tactile stimulations experiment to verify the optimal resonance frequency of human's tactile system. In: 2015 IEEE International Conference on Information and Automation, 2015, S. 2960-2964

[47] Weinstein, S.: Invitational Lecture: Fifty years of somatosensory research. In: Journal of Hand Therapy 6 (1993), Nr. 1, 11-22. http://dx.doi.org/10. 1016/S0894-1130(12)80176-1. - DOI 10.1016/S08941130(12)80176-1. - ISSN 0894-1130

[48] Yao, L. ; Meng, J. ; Zhang, D. ; Sheng, X. ; Zhu, $\mathrm{X}$. .: Combining motor imagery with selective sensation toward a hybrid-modality BCI. In: IEEE transactions on bio-medical engineering 61 (2014), Nr. 8, S. 23042312. http://dx.doi.org/10.1109/TBME. 2013.2287245. - DOI 10.1109/TBME.2013.2287245. - ISSN 1558-2531

[49] Zhou, L. ; TaO, X. ; He, F. ; Zhou, P. ; QI, H.: Reducing false triggering caused by irrelevant mental activities in brain-computer interface based on motor imagery. In: IEEE journal of biomedical and health informatics PP (2021). http://dx.doi.org/10.1109/ JBHI . 2021.3066610. - DOI 10.1109/JBHI.2021.3066610 - ISSN 2168-2208 